\title{
COLING 2020
}

\section{The 27th International Conference on Computational Linguistics}

Proceedings of System Demonstrations

December 8-13, 2020

Barcelona, Spain (Online) 
Copyright of each paper stays with the respective authors (or their employers).

ISBN 978-1-952148-28-6 


\section{Preface}

This volume contains papers from the system demonstration session of the 28th International Conference on Computational Linguistics (COLING 2020) held in online. Due to the COVID-19 pandemic the conference is held fully online under the auspices of the International Committee on Computational Linguistics (ICCL).

The demonstration session complements the conference's presentation and poster sessions and is focused on working software systems that are the tangible outcomes of research on computational linguistics.

As a result of a rigorous review process, we accepted 16 papers out of 55 submissions. The program committee consisted of 88 members and two chairs from both academia and industry. Each member evaluated 1-2 papers, which amounted to at least two reviews per paper. The acceptance criteria we followed during the selection process included the quality of work as well as the utility and demonstrability potential of the presented systems. Consequently, most of the accepted systems are user-interactive and feature rich graphical user interfaces.

First and foremost we would like to thank the program committee for their hard work and dedication to help make this event a success. Our special thanks also go to the people who made COLING 2020 and this volume possible. We thank General Chair, Donia Scott (University of Sussex), Program CoChairs, Nuria Bel (Pompeu Fabra University) and Chengqing Zong (Chinese Academy of Sciences), Local Organization Co-Chairs, Leo Wanner (Pompeu Fabra University), Horacio Saggion (Pompeu Fabra University), Mónica Domínguez (Pompeu Fabra University), Publication Co-Chairs, Derek Wong (University of Macau), Liang Huang (Oregon State University), Yang Zhao (Chinese Academy of Sciences), Virtual Infrastructure Co-Chairs, Paul Piwek (The Open University), Lluís Padró Cirera (Polytechnic University of Catalonia), Luis Espinosa Anke (Cardiff University), Publicity Co-Chairs, Ghazaleh Kazeminejad (University of Colorado Boulder), Tiejun Zhao (Harbin Institute of Technology), Ted Pedersen (University of Minnesota), Anna Rogers (University of Copenhagen), Sponsorship CoChairs, Feiyu Xu (SAP), Alexander Löser (Beuth University of Applied Sciences Berlin), Jose Manuel Gómez Pérez (Expert System Iberia), Virtual Social Chair, Esther Seyffarth (Heinrich Heine University), Web Co-Chairs, Laura Pérez-Mayos (Pompeu Fabra University), Amita Misra (IBM Watson), Ethics Advisory Group, Tim Baldwin (University of Melbourne), Amanda Stent (Bloomberg LP), Emily Bender (University of Washington), Dirk Hovy (Bocconi University), Ted Pedersen (University of Minnesota), Pascale Fung (Hong Kong University of Science and Technology), Saif M. Mohammad (National Research Council Canada), and our Local Support, Joana Clotet (Pompeu Fabra University) for their tireless work.

Michal PTASZYNSKI and Bartsz ZIOLKO

COLING 2020 Demonstration Program Co-Chairs

07 November 2020 



\section{Demonstration Co-Chairs:}

Michal Ptaszynski, Kitami Institute of Technology

Bartosz Ziolko, Techmo

\section{Program Committee:}

Lars Ahrenberg, Linköping University

Eiji Aramaki, NAIST

Elizabeth Boschee, Information Sciences Institute

Yi Cai, South China University of Technology

Vittorio Castelli, IBM Research AI

Angel Chang, Simon Fraser University

Nancy Chen, Institute for Infocomm Research, A*STAR

Zhumin Chen, Shandong University

Li Cheng, Xinjiang Technical Institute of Physics \& Chemistry, Chinese Academy of Sciences

Christian Chiarcos, Goethe-Universität Frankfurt am Main

Christos Christodoulopoulos, Amazon Research

Danilo Croce, University of Roma, Tor Vergata

Patrick Ehlen, Loop AI Labs

Michael Elhadad, Ben Gurion University

Andrea Esuli, ISTI-CNR

Yang Feng, Institute of Computing Technology, Chinese Academy of Sciences

Chong Feng, Beijing Institute of Technology

Radu Florian, IBM Research

Dimitris Galanis, Institute for Language and Speech Processing, Athena Research Center

Michael Gamon, Microsoft Research

Siva Reddy Gangireddy, Voysis

Roxana Girju, University of Illinois, Urbana-Champaign

Xianpei Han, Institute of Software, Chinese Academy of Sciences

Tianyong Hao, School of Computer Science, South China Normal University

Kenji Hatano, Doshisha University

Yifan He, Alibaba Group

Ales Horak, Masaryk University

Magdalena Igras-Cybulska, AGH University of Science and Technology

Shajith Ikbal, IBM Research AI, India.

Iustina Ilisei, Cognizant Technology Solutions

Adam Jatowt, Kyoto University

Wenbin Jiang, Baidu Inc.

Mamoru Komachi, Tokyo Metropolitan University

Valia Kordoni, Humboldt-Universität zu Berlin

Marek Kubis, Adam Mickiewicz University

Carolin Lawrence, NEC Laboratories Europe

John Lee, City University of Hong Kong

Yves Lepage, Waseda University

Nikola Ljubešić, Jožef Stefan Institute

Wolfgang Maier, Mercedes-Benz AG

Benjamin Marie, NICT 
Stella Markantonatou, ILSP/R.C. "Athena"

Kazuya Mera, Hiroshima City University

Makoto Miwa, Toyota Technological Institute

Taesun Moon, IBM Research

Mikolaj Morzy, Poznan University of Technology

Philippe Muller, IRIT, University of Toulouse

Koji Murakami, Rakuten Institute of Technology NY

Preslav Nakov, Qatar Computing Research Institute, HBKU

Vincent $\mathrm{Ng}$, University of Texas at Dallas

Eric Nichols, Honda Research Institute Japan

Jagna Nieuwazny, Kitami Institute of Technology

Karol Nowakowski, Kitami Institute of Technology

Pierre Nugues, Lund University

Maciej Ogrodniczuk, Institute of Computer Science, Polish AcadeInstitute of Physicsmy of Sciences

Naoaki Okazaki, Tokyo Institute of Technology

Constantin Orasan, University of Surrey

Aasish Pappu, Spotify Research

Yannick Parmentier, University of Lorraine

Siddharth Patwardhan, Apple

Prokopis Prokopidis, ILSP/Athena RC

Guilin Qi, Southeast University

Likun Qiu, Minjiang University

Carlos Ramisch, Aix Marseille University, CNRS, LIS

German Rigau, UPV/EHU

Robert Ross, Technological University Dublin

Rafal Rzepka, Hokkaido University

Saurav Sahay, Intel Labs

Satoshi Sekine, Riken, AIP

Michel Simard, NRC

Vivek Srikumar, University of Utah

Mihai Surdeanu, University of Arizona

Kaveh Taghipour, Qritive

Christoph Teichmann, Bloomberg LP

Nadi Tomeh, LIPN, Université Sorbonne Paris Nord

Andrea Varga, CUBE

Yannick Versley, Amazon Alexa

Rui Wang, Alibaba Group

Aleksander Wawer, Institute of Computer Science, Polish Academy of Sciences

Guillaume Wisniewski, Université Paris and LLF

Kun Xu, Tencent AI Lab

Ruifeng Xu, Harbin Institute of Technology, Shenzhen

Tae Yano, Expedia Group

Motoki Yatsu, Aoyama Gakuin University

Dongdong Zhang, Microsoft Research Asia

Guangyou Zhou, School of Computer Science, Central China Normal University

Imed Zitouni, Google

Pierre Zweigenbaum, LIMSI, CNRS, Université Paris-Saclay 


\section{Table of Contents}

Ve'rdd. Narrowing the Gap between Paper Dictionaries, Low-Resource NLP and Community Involvement

Khalid Alnajjar, Mika Hämäläinen, Jack Rueter and Niko Partanen $\ldots \ldots \ldots \ldots \ldots \ldots \ldots \ldots 1$

MaintNet: A Collaborative Open-Source Library for Predictive Maintenance Language Resources

Farhad Akhbardeh, Travis Desell and Marcos Zampieri............................. 7

DART: A Lightweight Quality-Suggestive Data-to-Text Annotation Tool

Ernie Chang, Jeriah Caplinger, Alex Marin, Xiaoyu Shen and Vera Demberg .............. 12

Demo Application for the AutoGOAL Framework

Suilan Estevez-Velarde, Alejandro Piad-Morffis, Yoan Gutiérrez, Andres Montoyo, Rafael Muñoz-

Guillena and Yudivián Almeida Cruz .......................................... 18

Fast Word Predictor for On-Device Application

Huy Tien Nguyen, Khoi Tuan Nguyen, Anh Tuan Nguyen and Thanh Lac Thi Tran ......... 23

Semantic search with domain-specific word-embedding and production monitoring in Fintech

Mojtaba Farmanbar, Nikki Van Ommeren and Boyang Zhao ..................... 28

CogniVal in Action: An Interface for Customizable Cognitive Word Embedding Evaluation

Nora Hollenstein, Adrian van der Lek and Ce Zhang .......................... 34

A Multilingual Reading Comprehension System for more than 100 Languages

Anthony Ferritto, Sara Rosenthal, Mihaela Bornea, Kazi Hasan, Rishav Chakravarti, Salim Roukos,

Radu Florian and Avi Sil ............................................ 41

XplaiNLI: Explainable Natural Language Inference through Visual Analytics

Aikaterini-Lida Kalouli, Rita Sevastjanova, Valeria de Paiva, Richard Crouch and Mennatallah El-

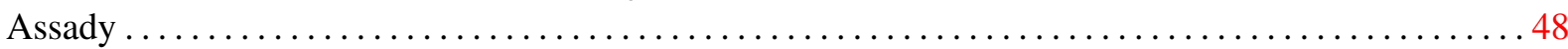

Discussion Tracker: Supporting Teacher Learning about Students' Collaborative Argumentation in High School Classrooms

Luca Lugini, Christopher Olshefski, Ravneet Singh, Diane Litman and Amanda Godley ...... 53

An Online Readability Leveled Arabic Thesaurus

Zhengyang Jiang, Nizar Habash and Muhamed Al Khalil .......................... 59

TrainX - Named Entity Linking with Active Sampling and Bi-Encoders

Tom Oberhauser, Tim Bischoff, Karl Brendel, Maluna Menke, Tobias Klatt, Amy Siu, Felix Alexander Gers and Alexander Löser ............................................. 64

BullStop: A Mobile App for Cyberbullying Prevention

Semiu Salawu, Yulan He and Jo Lumsden ................................. 70

Annobot: Platform for Annotating and Creating Datasets through Conversation with a Chatbot

Rafał Poświata and Michał Perełkiewicz ..................................... 75

Arabic Curriculum Analysis

Hamdy Mubarak, Shimaa Amer, Ahmed Abdelali and Kareem Darwish ................. 80 
Epistolary Education in 21st Century: A System to Support Composition of E-mails by Students to Superiors in Japanese

Kenji Ryu and Michal Ptaszynski....................................... 87 
December 9 (Wed), 2020, 16:00-16:30

Session 1 Tools for facilitating research and everyday use

MaintNet: A Collaborative Open-Source Library for Predictive Maintenance Language Resources

Farhad Akhbardeh, Travis Desell and Marcos Zampieri

DART: A Lightweight Quality-Suggestive Data-to-Text Annotation Tool

Ernie Chang, Jeriah Caplinger, Alex Marin, Xiaoyu Shen and Vera Demberg

Fast Word Predictor for On-Device Application

Huy Tien Nguyen, Khoi Tuan Nguyen, Anh Tuan Nguyen and Thanh Lac Thi Tran

XplaiNLI: Explainable Natural Language Inference through Visual Analytics

Aikaterini-Lida Kalouli, Rita Sevastjanova, Valeria de Paiva, Richard Crouch and Mennatallah El-Assady

An Online Readability Leveled Arabic Thesaurus

Zhengyang Jiang, Nizar Habash and Muhamed Al Khalil

TrainX - Named Entity Linking with Active Sampling and Bi-Encoders

Tom Oberhauser, Tim Bischoff, Karl Brendel, Maluna Menke, Tobias Klatt, Amy Siu, Felix Alexander

Gers and Alexander Löser

Annobot: Platform for Annotating and Creating Datasets through Conversation with a Chatbot

Rafał Poświata and Michał Perełkiewicz

Arabic Curriculum Analysis

Hamdy Mubarak, Shimaa Amer, Ahmed Abdelali and Kareem Darwish

Epistolary Education in 21st Century: A System to Support Composition of E-mails by Students to Superiors in Japanese

Kenji Ryu and Michal Ptaszynski

\section{December 11 (Fri), 2020, 16:00-16:30}

Session 2 Tools for NLP applications to other fields

Ve'rdd. Narrowing the Gap between Paper Dictionaries, Low-Resource NLP and Community Involvement

Khalid Alnajjar, Mika Hämäläinen, Jack Rueter and Niko Partanen

Demo Application for the AutoGOAL Framework

Suilan Estevez-Velarde, Alejandro Piad-Morffis, Yoan Gutiérrez, Andres Montoyo, Rafael Muñoz-Guillena and Yudivián Almeida Cruz

Semantic search with domain-specific word-embedding and production monitoring in Fintech Mojtaba Farmanbar, Nikki Van Ommeren and Boyang Zhao

CogniVal in Action: An Interface for Customizable Cognitive Word Embedding Evaluation

Nora Hollenstein, Adrian van der Lek and Ce Zhang 
A Multilingual Reading Comprehension System for more than 100 Languages

Anthony Ferritto, Sara Rosenthal, Mihaela Bornea, Kazi Hasan, Rishav Chakravarti, Salim Roukos, Radu Florian and Avi Sil

BullStop: A Mobile App for Cyberbullying Prevention

Semiu Salawu, Yulan He and Jo Lumsden

Discussion Tracker: Supporting Teacher Learning about Students' Collaborative Argumentation in High School Classrooms

Luca Lugini, Christopher Olshefski, Ravneet Singh, Diane Litman and Amanda Godley 